N. Obata

Nagoya Math. J.

Vol. 105 (1987), 109-119

\title{
CERTAIN UNITARY REPRESENTATIONS OF THE INFINITE SYMMETRIC GROUP, I
}

\author{
NOBUAKI OBATA
}

\section{Introduction}

Let $X$ be the set of all natural numbers and let $\widetilde{S}_{\infty}$ be the group of all finite permutations of $X$. The group $\widetilde{S}_{\infty}$, equipped with the discrete topology, is called the infinite symmetric group. It was discussed in F. J. Murray and J. von Neumann [3] as a concrete example of an ICC-group, which is a discrete group with infinite conjugacy classes. It is proved that the regular representation of an ICC-group is a factor representation of type $\mathrm{II}_{1}$. The infinite symmetric group is, therefore, a group not of type I. This may be the reason why its unitary representations have not been investigated satisfactorily. In fact, only few results are known. For instance, all indecomposable central positive definite functions on $\mathfrak{S}_{\infty}$, which are related to factor representations of type $\Pi_{1}$, were given by $\mathrm{E}$. Thoma [6]. Later on, A. M. Vershik and S. V. Kerov obtained the same result by a different method in [7] and gave a realization of the representations of type $\mathrm{II}_{1}$ in [8]. Concerning irreducible representations, A. Lieberman [2] and G. I. Ol'shanskii [4] obtained a characterization of a certain family of countably many irreducible representations by introducing a particular topology in $\mathfrak{S}_{\infty}$. However, irreducible representations have been studied not so actively as factor representations.

The main purpose of the present paper is to give a family of uncountably many irreducible representations of $\mathbb{S}_{\infty}$ explicitly with the həlp of induced representation. Let Aut $(X)$ denote the group of all bijections (or automorphisms) from $X$ onto itself. For any $\theta \in \operatorname{Aut}(X)$ we denote by $H(\theta)$ the subgroup of all permutations in $\widetilde{S}_{\infty}$ which commute with $\theta$. For each unitary character $\chi$ of $H(\theta)$ we form the induced representation $U^{\theta, x}=$ $\operatorname{Ind}_{H(\theta)}^{\Phi_{\infty}} \chi$ on $L^{2}\left(\Im_{\infty} / H(\theta)\right)$, the Hilbert space of all square summable functions on $\mathfrak{S}_{\infty} / H(\theta)$.

In this paper, for technical simplicity, we shall restrict ourselves to a special kind of automorphisms as follows. For each $p \geqslant 2$, we denote

Received September 13, 1985. 
by $\operatorname{Aut}_{p}(X)$ the set of all automorphisms $\theta$ having the next two properties:

(i) $\theta=\prod_{m=1}^{\infty}\left(i_{m 0} i_{m 1} \cdots i_{m p-1}\right)$ in cycle-notation;

(ii) $\operatorname{supp} \theta=X$, i.e. no point of $X$ is fixed by $\theta$.

As is shown in Section 2, the subgroup $H(\theta)$ is a semidirect product of an abelian group and the infinite symmetric group, and admits exactly $2 p$ unitary characters.

With the help of a general theory of unitary representations of discrete groups (see Section 1), we obtain the first result:

Theorem 1. Let $\theta$ be a member of $\operatorname{Aut}_{p}(X)$ with $p \geqslant 2$.

(1) For any unitary character $\chi$ of $H(\theta), U^{\theta, \chi}$ is irreducible.

(2) For two unitary characters $\chi$ and $\chi^{\prime}$ of $H(\theta), U^{\theta, \chi}$ is equivalent to $U^{\theta, \chi^{\prime}}$ if and only if $\chi=\chi^{\prime}$.

Next we shall discuss equivalence between two irreducible representations $U^{\theta, x}$ and $U^{\theta^{\prime}, x^{\prime}}$. For two automorphisms $\theta=\prod_{m=1}^{\infty}\left(i_{m 0} i_{m 1} \cdots i_{m p-1}\right)$ and $\theta^{\prime}=\prod_{n=1}^{\infty}\left(j_{n 0} j_{n 1} \cdots j_{n p-1}\right)$ in $\operatorname{Aut}_{p}(X)$, we denote by $N\left(\theta, \theta^{\prime}\right)$ the number of pairs $(m, n)$ such that $\left\{i_{m 0}, \cdots, i_{m p-1}\right\}=\left\{j_{n 0}, \cdots, j_{n p-1}\right\}$.

TheOREM 2. Let $\theta$ and $\theta^{\prime}$ be members of $\operatorname{Aut}_{p}(X)$ and $\operatorname{Aut}_{p^{\prime}}(X)$ with $p, p^{\prime} \geqslant 2$, and let $\chi$ and $\chi^{\prime}$ be unitary characters of $H(\theta)$ and $H\left(\theta^{\prime}\right)$, respectively.

(1) If $p=p^{\prime}$ and if $N\left(\theta, \theta^{\prime}\right)$ is finite, $U^{\theta, x}$ is not equivalent to $U^{\theta^{\prime}, x^{\prime}}$.

(2) If $p \neq p^{\prime}, U^{\theta, x}$ is not equivalent to $U^{\theta^{\prime}, x^{\prime}}$.

Finally we refer to the irreducible representations discussed in [2] and [4]. Let $\rho$ be an irreducible representation of the finite symmetric group $\mathfrak{S}_{s}, s=0,1,2, \cdots$. We denote by $\pi^{\circ}$ the representation of $\mathfrak{\varsigma}_{\infty}$ corresponding to $\rho$. By a slight modification we obtain another class of irreducible representations of $\widetilde{S}_{\infty}$, which are denoted by $\bar{\pi}^{o}$. (For details, see Section 3.) We have the following

THEOREM 3. Let $\theta$ be a member of $\operatorname{Aut}_{p}(X)$ with $p \geqslant 2$ and let $\chi$ be a unitary character of $H(\theta)$. Then $U^{\theta, x}$ is equivalent to neither $\pi^{\rho}$ nor $\bar{\pi}^{\rho}$ for any irreducible representation $\rho$ of $\widetilde{\varsigma}_{s}, s=0,1,2, \cdots$.

For an arbitrary automorphism $\theta \in \operatorname{Aut}(X)$, the unitary representation $U^{\theta, x}$ is not irreducible in general. If $\theta$ has a finite support, i.e. $\theta \in \mathbb{S}_{\infty}$, the corresponding unitary representation is decomposed into a sum of irreducible ones $\pi^{o}$ and $\bar{\pi}^{\rho}$. Hence our method yields the irreducible repre- 
sentations discussed in [2] and [4]. It would be possible to discuss a general case with our technique.

The author would like to express his thanks to Professor H. Yoshizawa for his kind advice.

\section{$\S 1$. Preliminary results}

Let $G$ be a discrete group, $H$ a subgroup and $\Omega=G / H$ the quotient space. We denote by $\omega_{0} \in \Omega$ the point whose isotropy group is $H$. For each unitary character $\chi$ of $H$ we consider the induced representation $U^{x}$ $=\operatorname{Ind}_{H}^{G} \chi$. It is convenient to adopt the following realization of $U^{\chi}$.

Let $L^{2}(\Omega)$ be the Hilbert space of all square summable functions on $\Omega$. We fix a cross section $\omega \mapsto s[\omega] \in G$ for the canonical projection $g \mapsto g \omega_{0}$ $\in \Omega, g \in G$. Then the induced representation $U^{x}$ is given by the formula:

$$
\left(U^{\chi}(g) f\right)(\omega)=\chi\left(s[\omega]^{-1} g s\left[g^{-1} \omega\right]\right) f\left(g^{-1} \omega\right),
$$

where $f \in L^{2}(\Omega)$ and $g \in G$. We may assume $s\left[\omega_{0}\right]=e$ (the identity).

For each $\omega \in \Omega$ we denote by $\delta_{\omega}$ the delta-function concentrated at $\omega$, namely, $\delta_{\omega}\left(\omega^{\prime}\right)=1$ if $\omega=\omega^{\prime}$ and $=0$ otherwise. Then the family $\left\{\delta_{\omega}\right.$; $\omega \in \Omega\}$ becomes a complete orthonormal basis for $L^{2}(\Omega)$. For any $g \in G$ we have

$$
U^{\chi}(g) \delta_{\omega_{0}}=\chi\left(s\left[g \omega_{0}\right]^{-1} g\right) \delta_{g \omega_{0}} .
$$

Here we note that the factor $\chi\left(s\left[g \omega_{0}\right]^{-1} g\right)$ is a constant and that $\delta_{\omega_{0}}$ is a cyclic vector for the unitary representation $U^{*}$.

Proposition 1.1. Assume that all H-orbits in $\Omega$ are infinite sets except the orbit $\left\{\omega_{0}\right\}$. Then we have

(1) $U^{x}$ is irreducible;

(2) $U^{x}$ is equivalent to $U^{x^{\prime}}$ if and only if $\chi=\chi^{\prime}$.

Proof. (1) Suppose that $T$ is a bounded operator on $L^{2}(2)$ satisfying $U^{x}(g) T=T U^{x}(g)$ for all $g \in G$. If $h \in H$, we have

$$
U^{\chi}(h) T \delta_{\omega_{0}}=T U^{\chi}(h) \delta_{\omega_{0}}=\chi(h) T \delta_{\omega_{0}} .
$$

Therefore, in view of the definition of $U^{x(h)}$, we see that

$$
\left|T \delta_{\omega_{0}}\left(h^{-1} \omega\right)\right|=\left|T \delta_{\omega_{0}}(\omega)\right|, \quad h \in H, \quad \omega \in \Omega .
$$

Since $T \delta_{\omega_{0}} \in L^{2}(\Omega)$, it follows from the assumption that $T \delta_{\omega_{0}}=t \delta_{\omega_{0}}$ for some $t \in C$. Consequently, for any $g \in G$ we have 


$$
T U^{x}(g) \delta_{\omega_{0}}=U^{x}(g) T \delta_{\omega_{0}}=t U^{x}(g) \delta_{\omega_{0}},
$$

which implies $T=t I$ ( $I$ is the identity operator). By repeating the above proof we can show (2) easily.

If $\chi$ is a unitary character of $H$ and if $\alpha$ is an automorphism of $G$, we define a unitary character $\chi^{\alpha}$ of $\alpha(H)$ by

$$
\chi^{\alpha}(\alpha(h))=\chi(h), \quad h \in H .
$$

We put $V^{x}=V^{x, \alpha}=\operatorname{Ind}_{\alpha(H)}^{G} \chi^{\alpha}$. Using the natural isomorphism between $L^{2}(\Omega)=L^{2}(G / H)$ and $L^{2}(G / \alpha(H))$, we can realize $V^{x}$ on $L^{2}(\Omega)$ :

$$
\left(V^{\chi}(\alpha(g)) f\right)(\omega)=\chi\left(s[\omega]^{-1} g s\left[g^{-1} \omega\right]\right) f\left(g^{-1} \omega\right),
$$

where $f \in L^{2}(\Omega)$ and $g \in G$. In other words, $U^{x}=V^{x} \circ \alpha$, where $U^{x}=$ $\operatorname{Ind}_{H}^{G} \chi$ as before.

Proposition 1.2. If $\left|H: \alpha\left(g H g^{-1}\right) \cap H\right|=+\infty$ for all $g \in G$, two unitary representations $U^{\chi}$ and $V^{\chi^{\prime}}$ are disjoint for any unitary characters $\chi$ and $\chi^{\prime}$ of $H$.

Proof. Suppose that $T$ is a bounded operator on $L^{2}(\Omega)$ satisfying $T U^{x}(g)=V^{x^{\prime}}(g) T$ for all $g \in G$. If $h \in H$, we have

$$
V^{\chi^{\prime}}(h) T \delta_{\omega_{0}}=T U^{\chi}(h) \delta_{\omega_{0}}=\chi(h) T \delta_{\omega_{0}} .
$$

Hence

$$
\left|T \delta_{\omega_{0}}\left(\alpha^{-1}\left(h^{-1}\right) \omega\right)\right|=\left|T \delta_{\omega_{0}}(\omega)\right|, \quad h \in H, \quad \omega \in \Omega .
$$

On the other hand, the $\alpha^{-1}(H)$-orbit containing $g \omega_{0}(\in \Omega)$ is isomorphic to $\alpha^{-1}(H) / g H g^{-1} \cap \alpha^{-1}(H)$. Therefore all $\alpha^{-1}(H)$-orbits in $\Omega$ are infinite sets by assumption. Since $T \delta_{\omega_{0}} \in L^{2}(\Omega)$, we conclude $T \delta_{\omega_{0}}=0$. This implies $T=0$ immediately.

Remarks. (1) If the assumption of Proposition 1.2 holds, the automorphism $\alpha$ is necessarily an outer automorphism.

(2) Analoguous results are found in Godement [1] and Saito [5]. Yoshizawa [9] applied those arguments to free groups.

\section{$\S 2$. A characterization of certain subgroups of $\widetilde{S}_{\infty}$}

Let $X$ be the set of all natural numbers and $\widetilde{S}_{\infty}$ the group of all finite permutations of $X$. The group $\widetilde{\varsigma}_{\infty}$, equipped with the discrete topology, 
is called the infinite symmetric group. Each permutation $g \in \mathbb{S}_{\infty}$ can be written in cycle-notation, i.e. as a product of pairwise disjoint cycles.

We denote by $\operatorname{Aut}(X)$ the group of all bijections (or automorphisms) from $X$ onto itself. Obviously $\widetilde{S}_{\infty}$ is a normal subgroup of Aut $(X)$. Any $\theta \in \operatorname{Aut}(X)$ also admits a cycle-notation which may be an infinite product of cycles or may contain cycles of infinite length.

For any $\theta \in \operatorname{Aut}(X)$ we denote by $H(\theta)$ the subgroup of all permutations in $\widetilde{S}_{\infty}$ which commute with $\theta$ :

$$
H(\theta)=\left\{g \in \widetilde{S}_{\infty} ; g \theta=\theta g\right\}\left(=H\left(\theta^{-1}\right)\right) .
$$

In what follows we shall restrict ourselves to some special automorphisms of $X$. For any integer $p \geqslant 2$, we denote by $\operatorname{Aut}_{p}(X)$ the set of all automorphisms $\theta \in \operatorname{Aut}(X)$ having the following two properties:

(i) $\theta=\prod_{m=1}^{\infty}\left(i_{m 0} i_{m 1} \cdots i_{m p-1}\right)$ in cycle-notation;

(ii) $\operatorname{supp} \theta=X$, i.e. no point of $X$ is fixed by $\theta$.

Let $A(\theta)$ be the abelian subgroup of $\widetilde{S}_{\infty}$ which is generated by all cyclic permutations $\left(i_{m 0} i_{m 1} \cdots i_{m p-1}\right), m=1,2, \cdots$, and $S(\theta)$ the subgroup of all permutations $g \in \mathfrak{S}_{\infty}$ having the following property: there exists some $\sigma \in \mathfrak{S}_{\infty}$ such that $g\left(i_{m k}\right)=i_{\sigma(m) k}$ for all $m=1,2, \cdots$ and $k=0,1, \cdots p-1$. As is easily seen, $A(\theta) S(\theta)=S(\theta) A(\theta)=S(\theta) \ltimes A(\theta)$ (semidirect product) and $S(\theta) A(\theta) \subset H(\theta)$. Note that $S(\theta) A(\theta)$ does not depend on the choice of a sequence $\left\{i_{m 0}\right\}_{m=1,2, \ldots}$ though $S(\theta)$ does. The main purpose of this section is to show the following

Proposition 2.1. We have

$$
H(\theta)=S(\theta) \ltimes A(\theta) \quad \text { (semidirect product }) .
$$

We need some preliminaries. The group $A(\theta)$ is isomorphic to the restricted direct product group $\left(\boldsymbol{Z}_{p}\right)_{0}^{\infty}=\left\{a=\left(a_{1}, a_{2}, \cdots\right) \in\left(\boldsymbol{Z}_{p}\right)^{\infty} ; a_{n}=0\right.$ except finitely many $n\}$. On the other hand, $S(\theta)$ is isomorphic to $\widetilde{S}_{\infty}$ by definition. Through these isomorphisms we define a permutation $(\sigma, a)$ of $X$ by

$$
(\sigma, a)\left(i_{m k}\right)=i_{\sigma(m) k+a_{m}} .
$$

The second suffix $k+a_{m}$ is taken as an element of $\boldsymbol{Z}_{p}$. Then we have

$$
(\sigma, a)\left(\sigma^{\prime}, a^{\prime}\right)=\left(\sigma \sigma^{\prime}, a^{\sigma^{\prime}}+a^{\prime}\right),
$$

where $a^{\sigma^{\prime}}=\left(a_{\sigma^{\prime}(1)}, a_{\sigma^{\prime}(2)}, \cdots\right)$. 
Lemma 2.2. (1) Let $\sigma=\left(m_{1} m_{2} \cdots m_{n}\right) \in \mathbb{S}_{\infty}$ and $a=\left(a_{1}, a_{2}, \cdots\right) \in\left(Z_{p}\right)_{0}^{\infty}$. If $a_{j}=0$ for $j \neq m_{1}, \cdots, m_{n}$ and $a_{m_{1}}+a_{m_{2}}+\cdots+a_{m_{n}}=0(\bmod p)$, the cycle-notation of $(\sigma, a)$ is given by

$$
(\sigma, a)=\left(x_{1} \cdots x_{n}\right)\left(\theta\left(x_{1}\right) \cdots \theta\left(x_{n}\right)\right) \cdots\left(\theta^{p-1}\left(x_{1}\right) \cdots \theta^{p-1}\left(x_{n}\right)\right),
$$

where $x_{1}=i_{m_{1} 0}, x_{2}=i_{m_{2} a_{m_{1}}}, \cdots, x_{n}=i_{m_{n} a_{m_{1}}+\cdots+a_{m_{n-1}}}$.

(2) For $x_{1}, \cdots, x_{n} \in X$ we put $\gamma_{0}=\left(x_{1} x_{2} \cdots x_{n}\right)$ and $\gamma_{j}=\theta^{j} \gamma_{0} \theta^{-j}, j=$ $0,1, \cdots, p-1$. If $\gamma_{0}, \gamma_{1}, \cdots, \gamma_{p-1}$ are pairwise disjoint cycles, there exist $\sigma \in \mathbb{S}_{\infty}$ and $a \in\left(Z_{p}\right)_{0}^{\infty}$ such that $\gamma_{0} \gamma_{1} \ldots \gamma_{p-1}=(\sigma, a)$.

Proof. (1) If $m \neq m_{1}, \cdots, m_{n}$, obviously we have $(\sigma, a)\left(i_{m k}\right)=i_{m k}$. On the other hand, inductively we see

$$
\begin{aligned}
& (\sigma, a)\left(x_{1}\right)=(\sigma, a)\left(i_{m_{1}}\right)=i_{\sigma\left(m_{1}\right) a_{m_{1}}}=i_{m_{2} a_{m_{1}}}=x_{2}, \\
& (\sigma, a)^{2}\left(x_{1}\right)=(\sigma, a)\left(x_{2}\right)=i_{m_{3} a_{m_{1}}+a_{m_{2}}}=x_{3}, \\
& \quad \vdots \\
& (\sigma, a)^{n-1}\left(x_{1}\right)=(\sigma, a)\left(x_{n-1}\right)=i_{m_{n} a_{m_{1}}+\cdots+a_{m_{n-1}}}=x_{n}, \\
& (\sigma, a)^{n}\left(x_{1}\right)=i_{m_{1} a_{m_{1}}+\cdots+a_{m_{n}}}=i_{m_{1} 0}=x_{1} .
\end{aligned}
$$

Therefore the cycle $\left(x_{1} \cdots x_{n}\right)$ is contained in the cycle-notation of $(\sigma, a)$. Since $(\sigma, a)$ commutes with $\theta$, we obtain the desired expression.

(2) Choose $m_{1}, \cdots, m_{n} \geqslant 1$ and $0 \leqslant k_{1}, \cdots, k_{n}<p$ such that $x_{1}=i_{m_{1} k_{1}}$, $\cdots, x_{n}=i_{m_{n} k_{n}}$ and put $a_{m_{1}}=k_{2}-k_{1}, a_{m_{2}}=k_{3}-k_{2}, \cdots, a_{m_{n-1}}=k_{n}-k_{n-1}$, $a_{m_{n}}=k_{1}-k_{n}$. Since $m_{1}, \cdots, m_{n}$ are pairwise distinct by assumption, $\sigma=$ $\left(m_{1} \cdots m_{n}\right) \in \Im_{\infty}$. We define $a=\left(a_{1}, a_{2}, \cdots\right) \in\left(Z_{p}\right)_{0}^{\infty}$ by putting $a_{j}=0$ for all $j \neq m_{1}, m_{2}, \cdots, m_{n}$. Then it is easily seen that $\gamma_{0} \gamma_{1} \cdots \gamma_{p-1}=(\sigma, a)$.

Lemma 2.3. Let $q$ and $q^{\prime}$ be two positive integers. For $x_{1}, \cdots, x_{n} \in X$ we put (formally)

$$
\gamma_{0}=\left(x_{1} \cdots x_{n} \theta^{q}\left(x_{1}\right) \cdots \theta^{q}\left(x_{n}\right) \cdots \theta^{\left(q^{\prime}-1\right) q}\left(x_{1}\right) \cdots \theta^{\left(q^{\prime}-1\right) q}\left(x_{n}\right)\right) .
$$

(1) If $(p, q)=1$ and if $q^{\prime}=p, \gamma_{0}$ belongs to $S(\theta) A(\theta)$ whenever $\gamma_{0}$ is a cycle, i.e. all elements in the right hand side are mutually distinct.

(2) If $(p, q)=r \neq 1$ and if $q^{\prime}$ is the smallest positive integer such that $q q^{\prime}=0(\bmod p), \gamma_{0} \gamma_{1} \ldots \gamma_{r-1}$ belongs to $S(\theta) A(\theta)$ whenever $\gamma_{0}, \gamma_{1}, \cdots, \gamma_{r-1}$ are pairwise disjoint cycles, where $\gamma_{j}=\theta^{j} \gamma_{0} \theta^{-j}, j=0,1, \cdots, r-1$.

Proof. We put

$$
h=\left(x_{1} \theta\left(x_{1}\right) \cdots \theta^{p-1}\left(x_{1}\right)\right)^{q}\left(x_{1} \cdots x_{n}\right)\left(\theta\left(x_{1}\right) \cdots \theta\left(x_{n}\right)\right) \cdots\left(\theta^{p-1}\left(x_{1}\right) \cdots \theta^{p-1}\left(x_{n}\right)\right) .
$$


(1) In view of Lemma 2.2 one can easily verify that $h$ belongs to $S(\theta) A(\theta)$ and that $h=\gamma_{0}$.

(2) Obviously $h$ belongs to $S(\theta) A(\theta)$ and we see $h=\gamma_{0} \gamma_{1} \ldots \gamma_{r-1}$.

Proof of Proposition 2.1. We have only to show that $S(\theta) A(\theta) \supset H(\theta)$. Let $g$ be an arbitrary element of $H(\theta)$ and $g=g_{1} g_{2} \cdots g_{n}$, where $g_{k}=$ $\left(x_{k 1} x_{k 2} \cdots x_{k s_{k}}\right)$, its cycle-notation. For each $k=1,2, \cdots, n$, there exists a unique $l=l(k)$ such that $\theta g_{k} \theta^{-1}=g_{l}$, namely, $\left(\theta\left(x_{k 1}\right) \cdots \theta\left(x_{k s_{k}}\right)\right)=\left(x_{l 1} \cdots x_{l s_{l}}\right)$. Without loss of generality we may assume $k=1$. For simplicity we write $s_{1}=s$ and $x_{j}=x_{1 j}, j=1,2, \cdots, s$.

(a) In case of $l=1$, that is, $\left(\theta\left(x_{1}\right) \cdots \theta\left(x_{s}\right)\right)=\left(x_{1} \cdots x_{s}\right)$. As is easily seen, there exist two integers $t \geqslant 1$ and $q \geqslant 1$ with $(p, q)=1$ such that

$$
\left(x_{1} \cdots x_{s}\right)=\left(x_{1} \cdots x_{t} \theta^{q}\left(x_{1}\right) \cdots \theta^{q}\left(x_{t}\right) \cdots \theta^{(p-1) q}\left(x_{1}\right) \cdots \theta^{(p-1) q}\left(x_{t}\right)\right) \text {, }
$$

which belongs to $S(\theta) A(\theta)$ by Lemma 2.3 (1).

(b) In case of $l \neq 1$. There exists some $q$ with $1<q \leqslant p$ such that the cycles $\left(x_{1} \cdots x_{s}\right),\left(\theta\left(x_{1}\right) \cdots \theta\left(x_{s}\right)\right), \cdots,\left(\theta^{q-1}\left(x_{1}\right) \cdots \theta^{q-1}\left(x_{s}\right)\right)$ are pairwise disjoint but $\left(x_{1} \cdots x_{s}\right)=\left(\theta^{q}\left(x_{1}\right) \cdots \theta^{q}\left(x_{s}\right)\right)$. Necessarily $(p, q)=r \neq 1$ since $l \neq 1$. Let $q^{\prime}$ be the smallest positive integer such that $q q^{\prime}=0(\bmod p)$. By a similar argument to (a) we see that there exist two integers $t \geqslant 1$ and $u \geqslant 1$ with $\left(u, q^{\prime}\right)=1$ such that

$$
\left(x_{1} \cdots x_{s}\right)=\left(x_{1} \cdots x_{t} \theta^{q u}\left(x_{1}\right) \cdots \theta^{q u}\left(x_{t}\right) \cdots \theta^{\left(q^{\prime}-1\right) q u}\left(x_{1}\right) \cdots \theta^{\left(q^{\prime}-1\right) q u}\left(x_{t}\right)\right) .
$$

Since $(p, q u)=r,\left(x_{1} \cdots x_{s}\right)\left(\theta\left(x_{1}\right) \cdots \theta\left(x_{s}\right)\right) \cdots\left(\theta^{r-1}\left(x_{1}\right) \cdots \theta^{r-1}\left(x_{s}\right)\right)$ belongs to $S(\theta) A(\theta)$ by Lemma $2.3(2)$.

We end this section by giving the structure of $H(\theta)$ for a general automorphism $\theta \in \operatorname{Aut}(X)$. For any subset $Y$ of $X$ we denote by $\mathfrak{S}(Y)$ the subgroup of all permutations in $\subseteq_{\infty}$ which act identically outside $Y$. Let $\theta \in \operatorname{Aut}(X)$ be an automorphism whose cycle-notation is of the form: $\theta=$ $\prod_{m}\left(i_{m 0} i_{m 1} \cdots i_{m p-1}\right)$, where the number of the cycles is finite or infinite, and possibly $\operatorname{supp} \theta \neq X$. We denote by $H^{\prime}(\theta)$ the subgroup of all permutations in $\widetilde{S}(\operatorname{supp} \theta)$ which commute with $\theta$. Then the structure of $H^{\prime}(\theta)$ is known by virtue of Proposition 2.1. There is no difficulty in verifying the next result which describes the structure of $H(\theta)$ for an arbitrary automorphism $\theta \in \operatorname{Aut}(X)$.

Proposition 2.4. Any $\theta \in \operatorname{Aut}(X)$ admits an expression of the form: $\theta=\theta_{\infty} \theta_{2} \theta_{3} \cdots$, where $\theta_{n}$ is a product of disjoint cycles of length $n$ and the 
subsets $\operatorname{supp} \theta_{n}, n=\infty, 2,3, \cdots$ are mutually disjoint. Furthermore $H(\theta)=$ $\Im(X-\operatorname{supp} \theta) \times H^{\prime}\left(\theta_{2}\right) \times H^{\prime}\left(\theta_{3}\right) \times \cdots$ in the sense of restricted direct product.

\section{§3. Construction of irreducible representations}

We keep the notations introduced in the previous section. Let $\theta=$ $\prod_{m=1}^{\infty}\left(i_{m 0} i_{m 1} \cdots i_{m p-1}\right)$ be the cycle-notation of an automorphism $\theta \in \operatorname{Aut}_{p}(X)$. Since $S(\theta)$ is isomorphic to $\mathfrak{S}_{\infty}$, it has exactly two unitary characters: 1 (the trivial character) and $\operatorname{sgn}\left(S(\theta) \simeq \mathfrak{S}_{\infty} \ni \sigma \mapsto \operatorname{sgn} \sigma \in\{ \pm 1\}\right.$ ). For any $j=0,1, \cdots p-1$, we define a unitary character $\chi_{j}$ of $A(\theta) \simeq\left(Z_{p}\right)_{0}^{\infty}$ by

$$
\chi_{j}(a)=\exp \left(\frac{2 \pi j \sqrt{-1}}{p} \sum_{k=1}^{\infty} a_{k}\right), \quad a=\left(a_{1}, a_{2}, \cdots\right) .
$$

Then one can easily verify that $H(\theta)=S(\theta) A(\theta)$ has exactly $2 p$ unitary characters: $\chi_{j}^{+}=1 \otimes \chi_{j}$ and $\chi_{j}^{-}=\operatorname{sgn} \otimes \chi_{j}, j=0,1, \cdots, p-1$.

For any unitary character $\chi$ of $H(\theta)$ we put $U^{\theta, \chi}=\operatorname{Ind}_{H(\theta)}^{\Phi_{\infty}} \chi$. As in Section 1 , we put $\Omega=\mathfrak{S}_{\infty} / H(\theta)$ and $\omega_{0}$ denotes the point of $\Omega$ whose isotropy group is $H(\theta)$.

Lemma 3.1. All $H(\theta)$-orbits in $\Omega$ are infinite sets except $\left\{\omega_{0}\right\}$.

Proof. It is sufficient to show that $S(\theta) g \omega_{0}$ is an infinite set for any $g \notin H(\theta)$. Since $g$ does not commute with $\theta$, there exists some $n_{0} \in X$ such that $g^{-1} \theta\left(n_{0}\right) \neq \theta g^{-1}\left(n_{0}\right)$. Fix a sufficiently large $m_{0} \in X$ such that $\left\{n_{0}, \theta\left(n_{0}\right)\right.$, $\left.\cdots, \theta^{p-1}\left(n_{0}\right)\right\} \cup \operatorname{supp} g \subset \bigcup_{m=1}^{m_{0}}\left\{i_{m 0}, \cdots, i_{m p-1}\right\}$. Since $\theta^{q}\left(n_{0}\right) \in\left\{i_{10}, i_{20}, \cdots, i_{m_{0} 0}\right\}$ for some $q \geqslant 0, \sigma_{k}=\prod_{j=0}^{p-1}\left(\theta^{q+j}\left(n_{0}\right) i_{k j}\right)$ belongs to $S(\theta)$. It is sufficient to show that $\sigma_{k} g \omega_{0} \neq \sigma_{k^{\prime}} g \omega_{0}$ whenever $k \neq k^{\prime}>m_{0}$. In fact we see

$$
g^{-1} \sigma_{k^{\prime}}^{-1} \sigma_{k} g\left(i_{k^{\prime} p-q}\right)=g^{-1} \sigma_{k^{\prime}}^{-1}\left(i_{k^{\prime} p-q}\right)=g^{-1}\left(n_{0}\right)
$$

and

$$
g^{-1} \sigma_{k^{\prime}}^{-1} \sigma_{k} g\left(i_{k^{\prime} p-q+1}\right)=g^{-1} \sigma_{k^{\prime}}^{-1}\left(i_{k^{\prime} p-q+1}\right)=g^{-1}\left(\theta\left(n_{0}\right)\right) .
$$

Hence by assumption we obtain

$$
\theta g^{-1} \sigma_{k^{\prime}}^{-1} \sigma_{k} g\left(i_{k^{\prime} p-q}\right) \neq g^{-1} \sigma_{k^{\prime}}^{-1} \sigma_{k} g\left(i_{k^{\prime} p-q+1}\right)=g^{-1} \sigma_{k^{\prime}}^{-1} \sigma_{k} g \theta\left(i_{k^{\prime} p-q}\right) .
$$

This shows that $g^{-1} \sigma_{k^{\prime}}^{-1} \sigma_{k} g$ does not commute with $\theta$. Therefore we have $\sigma_{k} g \omega_{0} \neq \sigma_{k^{\prime}} g \omega_{0}$ as desired.

In view of Proposition 1.1, immediately we have the following

TheOREm 1. Let $\theta$ be a member of $\operatorname{Aut}_{p}(X)$ with $p \geqslant 2$. 
(1) For any unitary character $\chi$ of $H(\theta), U^{\theta, \chi}$ is irreducible.

(2) For two unitary characters $\chi$ and $\chi^{\prime}$ of $H(\theta), U^{\theta, \chi}$ is equivalent to $U^{\theta, \chi^{\prime}}$ if and only if $\chi=\chi^{\prime}$.

We shall now discuss the question of equivalence between two irreducible representations $U^{\theta, x}$ and $U^{\theta^{\prime}, x^{\prime}}$, where $\theta \in \operatorname{Aut}_{p}(X)$ and $\theta^{\prime} \in \operatorname{Aut}_{p^{\prime}}(X)$. First we assume $p=p^{\prime}$. For two automorphisms $\theta=\prod_{m=1}^{\infty}\left(i_{m 0} i_{m 1} \cdots i_{m p-1}\right)$ and $\theta^{\prime}=\prod_{n=1}^{\infty}\left(j_{n 0} j_{n 1} \cdots j_{n p-1}\right)$ in $\operatorname{Aut}_{p}(X)$, we denote by $N\left(\theta, \theta^{\prime}\right)$ the number of pairs $(m, n)$ such that $\left\{i_{m 0}, i_{m 1}, \cdots, i_{m p-1}\right\}=\left\{j_{n 0}, j_{n 1}, \cdots, j_{n p-1}\right\}$.

LEMma 3.2. If $N\left(\theta, \theta^{\prime}\right)$ is finite, $\left|H(\theta): H(\theta) \cap H\left(\theta^{\prime}\right)\right|=+\infty$.

Proof. Let $m_{0}$ be the largest number such that $\left\{i_{m_{0} 0}, \cdots, i_{m_{0} p-1}\right\}=$ $\left\{j_{n 0}, \cdots, j_{n p-1}\right\}$ for some $n$. Put $g_{m}=\left(i_{m 0} \cdots i_{m p-1}\right) \in A(\theta) \subset H(\theta)$. It is sufficient to show that $\left\{g_{m}\left(H(\theta) \cap H\left(\theta^{\prime}\right)\right) ; m>m_{0}\right\}$ is an infinite set. Suppose that $g_{m^{\prime}}^{-1} g_{m} \in H(\theta) \cap H\left(\theta^{\prime}\right)$ for two distinct numbers $m$ and $m^{\prime}>m_{0}$. Note that $g_{m^{\prime}}^{-1} g_{m}$ is just a cycle-notation. Since $g_{m}$ does not commute with $\theta^{\prime}$ by assumption, we have $\theta^{\prime} g_{m^{\prime}}^{-1} \theta^{\prime-1}=g_{m}$ and $\theta^{\prime} g_{m} \theta^{\prime-1}=g_{m^{\prime}}^{-1}$ In particular, $g_{m}$ is uniquely determined by $g_{m}$ if it exists. This proves the assertion.

Lemma 3.3. If $p^{\prime}$ is not a divisor of $p,\left|H(\theta): H(\theta) \cap H\left(\theta^{\prime}\right)\right|=+\infty$ for any $\theta \in \operatorname{Aut}_{p}(X)$ and $\theta^{\prime} \in \operatorname{Aut}_{p^{\prime}}(X)$.

The proof is similar to that of Lemma 3.2. In case when $p^{\prime}$ is a divisor of $p$, the above result does not hold in general.

TheOREM 2. Let $\theta$ and $\theta^{\prime}$ be members of $\operatorname{Aut}_{p}(X)$ and $\operatorname{Aut}_{p^{\prime}}(X)$ with $p, p^{\prime} \geqslant 2$, and let $\chi$ and $\chi^{\prime}$ be unitary characters of $H(\theta)$ and $H\left(\theta^{\prime}\right)$, respectively.

(1) If $p=p^{\prime}$ and if $N\left(\theta, \theta^{\prime}\right)$ is finite, $U^{\theta, x}$ is not equivalent to $U^{\theta^{\prime}, x^{\prime}}$.

(2) If $p \neq p^{\prime}, U^{\theta, x}$ is not equivalent to $U^{\theta^{\prime}, x^{\prime}}$.

Proof. (1) There exists some $\alpha \in \operatorname{Aut}(X)$ such that $\theta^{\prime}=\alpha \theta \alpha^{-1}$. We denote by $\hat{\alpha}$ the automorphism of $\mathfrak{S}_{\infty}$ defined by $\hat{\alpha}(g)=\alpha g \alpha^{-1}, g \in \widetilde{S}_{\infty}$. Obviously we have $\hat{\alpha}(H(\theta))=H\left(\theta^{\prime}\right)$. Note that $N\left(\theta, g \theta^{\prime} g^{-1}\right)$ is finite for all $g \in \mathfrak{S}_{\infty}$ by assumption. Since

$$
\hat{\alpha}\left(g H(\theta) g^{-1}\right)=\alpha g H(\theta) g^{-1} \alpha^{-1}=H\left(\alpha g \theta g^{-1} \alpha^{-1}\right)=H\left(\alpha g \alpha^{-1} \theta^{\prime} \alpha g^{-1} \alpha^{-1}\right),
$$

it follows from Lemma 3.2 that $\left|H(\theta): \hat{\alpha}\left(g H(\theta) g^{-1}\right) \cap H(\theta)\right|=+\infty$. Then the desired result follows immediately from Proposition 1.2.

(2) The proof is modelled after Proposition 1.2. Here we only note 
that $\left|H(\theta): H(\theta) \cap H\left(g \theta^{\prime} g^{-1}\right)\right|=+\infty$ for all $g \in \widetilde{S}_{\infty}$ whenever $p^{\prime}>p$. This follows from Lemma 3.3.

Next we shall recall the irreducible representations discussed by Lieberman [2] and Ol'shanskii [4]. For brevity we write $\mathfrak{S}_{s}=\mathfrak{S}(\{1,2, \cdots, s\})$ and $\widetilde{S}_{\infty-s}=\mathfrak{S}(\{s+1, s+2, \cdots\})$. For any finite dimensional unitary repre-

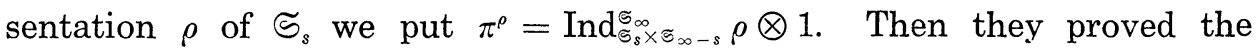
following

Proposition 3.4. (1) If $\rho$ is irreducible, so is $\pi^{\rho}$.

(2) Let $\rho$ and $\rho^{\prime}$ be irreducible representations of $\widetilde{S}_{s}$ and $\widetilde{S}_{s^{\prime}}$, respectively. Then $\pi^{\rho}$ is equivalent to $\pi^{\rho^{\prime}}$ if and only if $\rho$ is equivalent to $\rho^{\prime}$ (including $\left.s=s^{\prime}\right)$.

In addition we can construct another class of unitary representations of $\widetilde{\varsigma}_{\infty}$. For any finite dimensional unitary representation $\rho$ of $\mathfrak{S}_{s}$, we put $\bar{\pi}^{\rho}=\operatorname{Ind}_{\mathscr{\Xi}_{s} \times \Phi_{\infty}-s}^{\Phi_{\infty}} \rho \otimes \mathrm{sgn}$. The following result is then easily verified.

Proposition 3.5. (1) If $\rho$ is irreducible, so is $\bar{\pi}^{\rho}$.

(2) Let $\rho$ and $\rho^{\prime}$ be irreducible representations of $\mathfrak{S}_{s}$ and $\mathfrak{S}_{s^{\prime}}$, respectively. Then $\bar{\pi}^{\rho}$ is equivalent to $\bar{\pi}^{\rho^{\prime}}$ if and only if $\rho$ is equivalent to $\rho^{\prime}$.

(3) Let $\rho$ and $\rho^{\prime}$ be the same as above. Then $\bar{\pi}^{\rho}$ is not equivalent to $\pi^{\rho^{\prime}}$.

By repeating the proof of Proposition 1.2 we have the following result with no difficulty.

Theorem 3. Let $\theta$ be a member of $\operatorname{Aut}_{p}(X)$ with $p \geqslant 2$ and let $\chi$ be a unitary character of $H(\theta)$. Then $U^{\theta, x}$ is equivalent to neither $\pi^{\rho}$ nor $\bar{\pi}^{\rho}$ for any irreducible representation $\rho$ of $\widetilde{\varsigma}_{s}, s=0,1,2, \cdots$.

Remarks. (1) In this paper we restricted ourselves to rather special automorphisms $\theta \in \operatorname{Aut}_{p}(X)$ with $p \geqslant 2$ and discussed the corresponding unitary representations $U^{\theta, x}$. However, with the help of Proposition 2.4, we may discuss unitary representations corresponding to general automorphisms $\theta \in \operatorname{Aut}(X)$. Some comments for a particular case are given in the next paragraph.

(2) Let $\theta \in \operatorname{Aut}(X)$ have a finite support, i.e. $\theta \in \widetilde{S}_{\infty}$. By a suitable inner automorphism of $\mathfrak{S}_{\infty}$, we may assume $\operatorname{supp} \theta=\{1,2, \cdots, s\}$. Then $H(\theta)$ admits a direct product decomposition: $H(\theta)=H^{\prime}(\theta) \times \widetilde{\Im}_{\infty-s}$, with the notation introduced in Section 2. We now consider unitary representa- 
tions of $H(\theta)$ of the form $\rho^{\prime} \otimes 1$ and $\rho^{\prime} \otimes$ sgn, where $\rho^{\prime}$ is a finite dimensional unitary representation of $H^{\prime}(\theta)$. Note that all unitary characters of $H(\theta)$ are of the form above. Then we can prove the following result:

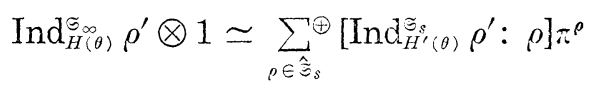

and

$$
\operatorname{Ind}_{H(\theta)}^{\Phi_{\infty}} \rho^{\prime} \otimes \operatorname{sgn} \simeq \sum_{\rho \in \hat{\bar{O}}_{s}}^{\oplus}\left[\operatorname{Ind} \tilde{H}_{H_{s}^{\prime}(\theta)}^{\tilde{s}_{s}} \rho^{\prime}: \rho\right] \bar{\pi}^{\rho},
$$

where $\hat{\Xi}_{s}$ denotes the set of all equivalence classes of irreducible representations of $\widetilde{\complement}_{s}$.

\section{REFERENCES}

[1] R. Godement, Les fonctions de type positif et la theorie des groupes, Trans. Amer. Math. Soc., 63 (1948), 1-84.

[2] A. Lieberman, The structure of certain unitary representations of infinite symmetric groups, Trans. Amer. Math. Soc., 164 (1972), 189-198.

[ 3 ] F. J. Murray and J. von Neumann, On rings of operators, IV, Ann. Math., 44 (1943), 716-808.

[ 4 ] G. I. Ol'shanskii, New "large" groups of type I, J. Soviet Math., 18 (1982), 22-39.

[5] M. Saito, Représentations unitaires monomiales d'un groupe discret, en particulier du groupe modulaire, J. Math. Soc. Japan, 26 (1974), 464-482.

[6] E. Thoma, Die unzerlegbaren, positiv-definiten Klassenfunktionen der abzählbar unendlichen, symmetrischen Gruppe, Math. Z., 85 (1964), 40-61.

[ 7 ] A. M. Vershik and S. V. Kerov, Asymptotic theory of characters of the symmetric group, Funct. Anal. Appl., 15 (1981), 246-255.

[8] - Characters and factor representations of the infinite symmetric group, Soviet Math. Dokl., 23 (1981), 389-392.

[ 9 ] H. Yoshizawa, Some remarks on unitary representations of the free group, Osaka Math. J., 3 (1951), 55-63.

Department of Mathematics

Faculty of Science

Nagoya University

Chikusa-ku, Nagoya 464

Japan 